\title{
Farelo de amêndoa da castanha de caju na alimentação de codornas japonesas na fase de postura ${ }^{1}$
}

\author{
Marcelo Borges Soares ${ }^{2}$, Maria de Fátima Freire Fuentes ${ }^{3}$, Ednardo Rodrigues Freitas ${ }^{3}$, Irani \\ Ribeiro Vieira Lopes $^{4}$, Rafaele Ferreira Moreira ${ }^{5}$, Francislene Silveira Sucupira ${ }^{5}$, Nádia de \\ Melo Braz ${ }^{5}$, Raffaella Castro Lima ${ }^{5}$
}

\footnotetext{
1 Parte da dissertação de Mestrado do primeiro autor apresentada ao curso de Pós-Graduação em Zootecnia/UFC

2 Eng. Agrônomo, MSc. em Zootecnia.

${ }^{3}$ Departamento de Zootecnia/CCA/UFC, Campus do Pici, Caixa Postal - 12.168, CEP: 60.355-970, Fortaleza, Ceará.

${ }^{4}$ Doutorado Integrado em Zootecnia, DZ/CCA/UFC.

${ }^{5}$ Graduação em Zootecnia/UFC. Bolsista do PIBIC, UFC/CNPq.
}

RESUMO - Avaliou-se o efeito da inclusão de farelo de amêndoa da castanha de caju (FACC) sobre a utilização dos nutrientes da dieta, o desempenho e as características dos ovos de codornas japonesas. Duzentos e setenta codornas com 17 semanas de idade foram pesadas e distribuídas em um delineamento inteiramente casualizado, com seis dietas e nove repetições de cinco aves. Foram testadas uma dieta controle (sem FACC) e cinco dietas com 4, 8, 12, 16 e 20\% de FACC. A digestibilidade da MS e do nitrogênio reduziu linearmente, entretanto, apenas com o nível de 20\% de FACC estes resultados foram menores que os obtidos com a dieta controle. A digestibilidade da gordura e da energia bruta e os valores de EM das dietas não foram influenciados pelos níveis de FACC na dieta. O consumo de ração não foi afetado pelos níveis desse alimento na dieta, mas a produção de ovos, o peso e a massa de ovo e a conversão alimentar decresceram linearmente com a inclusão de FACC na dieta. As porcentagens de albúmen e gema, assim como a coloração da gema, sofreram efeito quadrático dos níveis de FACC na dieta. A porcentagem de albúmen e a coloração da gema aumentaram e a porcentagem de gema reduziu com a inclusão de FACC em níveis superiores a $9 \%$. Em comparação à dieta controle, apenas a dieta com $20 \%$ de FACC prejudicou a produção de ovos, o peso e a massa de ovo, a coloração da gema e a conversão alimentar. O farelo de amêndoa da castanha de caju pode ser incluído em níveis de até 16\% em dietas para codornas japonesas em postura.

Palavras-chave: alimento alternativo, característica dos ovos, consumo de ração

\section{Cashew nut meal in laying diets for Japanese quails}

\begin{abstract}
This experiment was conducted to evaluate the effect of inclusion of cashew nut meal (CNM) in diets on nutrient digestibility, bird performance and egg characteristics of Japanese quails. A total of 270 japanese quails with 17 weeks of age were weighed and distributed into a completely randomized design with six diets and nine replicates of five birds in each experimental unit. Treatments consisted of one diet without CNM (control) and five diets containing 4; 8; 12; 16 and $20 \%$ of CNM. There was a linear reduction on digestibility coefficient of DM and nitrogen. But, significant difference in relation to control diet was detected only with diet containing $20 \%$ of CNM. Values of fat digestibility, gross energy and metabolizable energy of the diets were not affected by the inclusion levels of CNM. Feed intake was not affected by the inclusion of CNM. However egg production, egg weight, egg mass and feed conversion decreased linearly as dietary CNM levels increased in diets. Percentages of albumen and yolk as well as yolk color, showed a quadratic effect as dietary CNM levels increased. Albumen percentage and yolk color increased and yolk percentage decreased as CNM increased up to the level of 9\%. Comparing to control diet only treatment with 20\% CNM produced lower egg production, egg weight, egg mass, yolk color and bird feed conversion. In conclusion CNM can be included in Japanese quail diets in levels up to $16 \%$.
\end{abstract}

Key Words: alternative fed, egg characteristics, feed intake

\section{Introdução}

O aumento constante nos preços do milho e do farelo de soja tem levado a um crescente interesse por alimentos alternativos que possam ser utilizados em dietas para aves sem prejuízo ao desempenho desses animais.
No Ceará, entre os alimentos alternativos, destaca-se o farelo de amêndoa da castanha de caju (FACC), subproduto do beneficiamento da castanha. O FACC (Onifade et al., 1998; Onifade et al., 1999; Ojewola et al., 2004) é um subproduto de alto valor energético (6.306 a $6.764 \mathrm{kcal}$ de EB/kg de matéria natural) e protéico (22,15 a 38,12 \% de PB) 
e pode ser utilizado como substituto parcial ao milho e ao farelo de soja em dietas para aves. Segundo Onifade et al. (1998), o FACC pode ser comparado à soja integral e caracteriza-se como uma fonte moderada de proteína e uma excelente fonte de energia por apresentar elevado teor de gordura.

Os resultados de algumas pesquisas demonstraram a viabilidade do uso desse alimento em dietas para frangas de postura e frangos de corte. Onifade et al. (1998) constataram redução no consumo e melhora na conversão alimentar quando frangas na fase de recria foram alimentadas com dietas contendo FACC, resultado atribuído ao maior aproveitamento da energia da dieta contendo FACC. Militão (1999), Ojewola et al. (2004) e Freitas et al. (2006), em experimento com frangos de corte, verificaram que a inclusão de FACC nas dietas melhorou a conversão alimentar. Onifade et al. (1999) observaram que a inclusão de 31,5\% de FACC permitiu a utilização de subprodutos do milho e da casca de mandioca na dieta sem perda no desempenho de poedeiras.

A extrapolação de resultados obtidos em experimentos com poedeiras comerciais ou frangos de corte para codornas deve ser questionada, pois as diferenças anatômicas entre essas aves, em tamanho e comprimento dos órgãos do trato digestório, as particularidades fisiológicas e, às vezes, os hábitos alimentares, podem influenciar as respostas obtidas com um mesmo alimento em diferentes espécies de aves (Murakami \& Furlan, 2002). Assim, o objetivo nesta pesquisa foi avaliar os efeitos da inclusão de diferentes níveis de farelo de amêndoa da castanha de caju em dietas sobre a digestibilidade dos nutrientes, o desempenho e as características do ovo de codornas japonesas em postura.

\section{Material e Métodos}

Foram utilizadas 270 codornas japonesas com 17 semanas de idade distribuídas em um delineamento inteiramente casualizado, com seis tratamentos e nove repetições de cinco aves.

As aves foram alojadas em gaiolas de arame galvanizado (33 cm de comprimento $\times 23 \mathrm{~cm}$ de profundidade $\times$ $16 \mathrm{~cm}$ de altura), equipadas com bebedouro tipo nipple, comedouro tipo calha e coletor de ovos, cada uma com cinco aves.

No início do experimento, as aves foram pesadas individualmente e separadas por faixas de pesos com variação de $10 \mathrm{~g}$ entre os limites inferior e superior. Após a pesagem, as aves foram distribuídas para que todos os tratamentos tivessem repetições compostas de aves com pesos similares (peso médio $156 \pm 7,1 \mathrm{~g}$ ).

Os tratamentos consistiram de seis dietas: uma controle (sem FACC) e outras quatro, com 4, 8, 12, 16 e 20\%. As dietas experimentais (Tabela 1 ) foram formuladas considerando os valores de composição química e EM do FACC propostos pela tabela da Embrapa (1991), a composição dos demais ingredientes descrita por Rostagno et al. (2000) e as exigências nutricionais das codornas constantes no NRC (1994).

Para manter as dietas isoenergéticas, utilizaram-se óleo de soja e areia lavada como inerte para equilibrar os níveis de EM das dietas e, para ajustar os níveis de metionina, utilizou-se o aminoácido sintético DL - metionina.

O experimento teve 63 dias de duração, divididos em três períodos de 21 dias. Neste período, as aves receberam alimento e água à vontade. Para evitar desperdício, os comedouros foram abastecidos com as dietas duas vezes ao dia, às 8 e às $16 \mathrm{~h}$. O programa de luz utilizado foi de 16 horas de luz por dia (natural + artificial) e a coleta dos ovos foi realizada diariamente, às $9 \mathrm{~h}$, portanto, referente à produção do dia anterior.

Foram avaliados o consumo (g/ave/dia), a conversão alimentar (g/g), a porcentagem de postura (\%/ave/dia), o peso do ovo (g), a massa de ovo (g/ave/dia), a porcentagem de gema, casca e albúmen (\%) e a coloração da gema (leque colorimétrico da Roche).

No ensaio de metabolismo, foram instaladas bandejas cobertas com plástico sob as gaiolas de quatro repetições por tratamento. Para identificar as excretas provenientes da dieta fornecida durante o ensaio, foi adicionado $1 \%$ de óxido de ferro nas dietas no primeiro e no último dia de coleta. As excretas foram coletadas duas vezes ao dia (no início da manhã e ao final da tarde), acondicionadas em sacos plásticos, identificadas por repetição e congeladas.

Ao final do período experimental, foram determinadas a quantidade de alimento consumido e a quantidade total de excreta produzida. Após o descongelamento à temperatura ambiente, as excretas de cada repetição foram homogeneizadas para retirada de uma amostra, que foi encaminhada ao laboratório junto com amostras das dietas experimentais para determinação de MS, nitrogênio, EE e EM, segundo metodologia descrita por Silva \& Queiroz (2002). A EB foi determinada utilizando-se bomba calorimétrica adiabática, de acordo com Parr Instruments Co.

Com base nos resultados laboratoriais e utilizando-se as equações propostas por Matterson et al. (1965), foram calculados os coeficientes de digestibilidade aparente da MS (CDMS), do N (CDN) e do EE (CDEE) e os valores de 
Tabela 1 - Composições percentual e nutricional das dietas experimentais

Table 1 - Percentage and nutritional compositions of the experimental diets

\begin{tabular}{|c|c|c|c|c|c|c|}
\hline \multirow[t]{2}{*}{$\begin{array}{l}\text { Ingrediente } \\
\text { Ingredient }\end{array}$} & \multicolumn{6}{|c|}{$\begin{array}{c}\text { Nível de FACC (\%) } \\
\text { CNM level }\end{array}$} \\
\hline & 0 & 4 & 8 & 12 & 16 & 20 \\
\hline Farelo de soja (Soybean meal) & 33,340 & 31,580 & 29,81 & 28,60 & 27,560 & 26,510 \\
\hline FACC $(C N M)$ & 0,000 & 4,000 & 8,000 & 12,000 & 16,000 & 20,000 \\
\hline Calcário calcítico (Calcium limestone) & 5,624 & 5,635 & 5,648 & 5,654 & 5,658 & 5,660 \\
\hline Mistura vitamínica ${ }^{1}$ (Vitamin mix) $^{2}$ & 0,500 & 0,500 & 0,500 & 0,500 & 0,500 & 0,500 \\
\hline Mistura mineral $^{2}$ (Mineral mix) & 0,100 & 0,100 & 0,100 & 0,100 & 0,100 & 0,100 \\
\hline Sal (Salt) & 0,290 & 0,292 & 0,296 & 0,300 & 0,305 & 0,309 \\
\hline DL-metionina (DL-methionine) & 0,070 & 0,078 & 0,086 & 0,098 & 0,112 & 0,125 \\
\hline Antioxidante $^{3}$ (Antioxidant) & 0,020 & 0,020 & 0,020 & 0,020 & 0,020 & 0,020 \\
\hline Inerte $^{4}$ (Inert) & - & - & - & 1,430 & 3,286 & 5,167 \\
\hline $\mathrm{PB}(C P)(\%)$ & 20,00 & 20,00 & 20,00 & 20,00 & 20,00 & 20,00 \\
\hline Lisina (Lysine) (\%) & 1,097 & 1,083 & 1,069 & 1,063 & 1,060 & 1,056 \\
\hline Metionina + cistina (Methionine + cystine) $(\%)$ & 0,700 & 0,700 & 0,700 & 0,700 & 0,700 & 0,700 \\
\hline Metionina (Methionine) (\%) & 0,521 & 0,532 & 0,544 & 0,557 & 0,573 & 0,587 \\
\hline Treonina (Threonine) (\%) & 0,784 & 0,780 & 0,776 & 0,772 & 0,768 & 0,763 \\
\hline Triptofano (Tryptophan) (\%) & 0,268 & 0,270 & 0,272 & 0,275 & 0,278 & 0,282 \\
\hline $\mathrm{Ca}(\%)$ & 2,500 & 2,500 & 2,500 & 2,500 & 2,500 & 2,500 \\
\hline P disponível (\%) (Available P) & 0,350 & 0,350 & 0,350 & 0,350 & 0,350 & 0,350 \\
\hline Gordura (Fat) (\%) & 5,175 & 5,268 & 5,358 & 6,354 & 7,620 & 8,885 \\
\hline Ácido linoléico (\%) (Linoleic acid) & 2,808 & 2,885 & 2,960 & 3,516 & 4,215 & 4,914 \\
\hline $\mathrm{Na}(\%)$ & 0,150 & 0,150 & 0,150 & 0,150 & 0,150 & 0,150 \\
\hline
\end{tabular}

energia metabolizável aparente (EMA) e energia metabolizável aparente corrigida (EMAn).

A análise estatística dos dados foi realizada utilizando-se o SAS (2000) com um modelo inteiramente casualizado. Os graus de liberdade referentes aos níveis de farelo da amêndoa da castanha de caju, excluindo-se a ração testemunha (nível zero de inclusão do FACC), foram desdobrados em polinômios para estabelecer a curva que melhor descrevesse o comportamento dos dados.

Para comparação dos resultados obtidos com cada um dos níveis de inclusão do farelo da amêndoa da castanha de caju em relação à ração testemunha, foi utilizado o teste de Dunnett (5\%).

\section{Resultados e Discussão}

Os resultados obtidos no ensaio de metabolismo estão apresentados na Tabela 2. Segundo a análise de regressão, houve redução linear no CDMS ( $\hat{Y}=77,99-0,50 X$; $\left.r^{2}=42,04\right)$ eno $\operatorname{CDN}\left(\hat{Y}=50,42-0,71 X ; r^{2}=24,78\right)$, diminuindo em $0,50 \%$ a digestibilidade da MS e em 0,71\% a do nitrogênio para cada $1 \%$ de aumento na inclusão do FACC nas rações.

A comparação de médias pelo teste Dunnett (5\%) permitiu observar que apenas os coeficientes de digestibilidade da MS e do nitrogênio obtidos com a dieta com 20\% de FACC diferiram dos determinados com a dieta controle ( $0 \%$ de FACC). 
Segundo Militão (1999), o FACC é constituído de partes estragadas das amêndoas (com pintas pretas, manchas ou películas, ocasionadas pelo ataque de pragas ou doenças ou mesmo pelo processamento) ou de amêndoas inteiras. Sabe-se que o superaquecimento de um alimento pode promover a desnaturação da proteína comprometendo sua digestibilidade e, ainda, tornar alguns aminoácidos menos disponíveis, principalmente a lisina (Leeson \& Summers, 2001).

Diferentes amostras de FACC foram analisadas quanto à solubilidade da proteína em KOH, que variou de 89 a 96\%. Os menores valores de solubilidade foram determinados em amostras de FACC com grande proporção de pedaços de amêndoas com maior grau de tostagem. A menor solubilidade da proteína em $\mathrm{KOH}$ tem sido associada à menor digestibilidade da proteína e, conseqüentemente, dos aminoácidos da soja integral e do farelo de soja (Leeson \& Summers, 2001).

A redução do CDN das dietas com FACC está associada à qualidade da proteína desse alimento, pois sua inclusão diminuiu a quantidade de farelo de soja nas dietas (Tabela 1).

A digestibilidade do EE e da EB e os valores de EM das dietas não foram influenciados pelos níveis de FACC. Considerando que esse alimento é rico em EE e que a gordura foi igualmente digerida pelas aves de todos os tratamentos, é possível que o aumento da proporção de EE nas dietas com FACC tenha promovido a manutenção dos valores de EM das dietas, apesar da redução na digestibilidade da MS e do nitrogênio. De acordo com Sakomura et al. (2004), o efeito extrametabólico das gorduras melhora a eficiência energética, pois aumenta a energia líquida da dieta, em virtude de seu menor incremento calórico.
Onifade et al. (1998) atribuíram a redução no consumo de alimento e a melhora na conversão alimentar de frangas na fase de recria ao maior aproveitamento da energia das dietas contendo FACC.

Os resultados de desempenho obtidos com os diferentes níveis de FACC nas dietas são apresentados na Tabela 3. Considerando que a ingestão voluntária de alimento pelas codornas depende da ingestão de energia (Freitas et al., 2005), o fato de o consumo não ter variado significativamente entre os níveis de FACC pode ser atribuído à semelhante metabolização da energia das dietas (Tabela 2).

Outros autores (Militão, 1999; Ojewola et al., 2004; Freitas et al., 2006) verificaram que a inclusão de FACC em dietas para frangos de corte não influenciou o consumo de ração. Entretanto, Onifade et al. (1998) constataram redução no consumo quando frangas na fase de recria foram alimentadas com dietas contendo FACC. Segundo esses pesquisadores, essa resposta deveu-se ao maior aproveitamento da energia da dieta contendo FACC.

A produção de ovos diminuiu linearmente ( $\hat{Y}=95,04-$ 0,60 X; $\mathrm{r}^{2}=19,05 \%$ ) com o aumento dos níveis de FACC e, pelo teste de Dunnett (5\%), apenas nas aves alimentadas com a dieta com $20 \%$ de FACC a produção foi significativamente inferior à obtida com a dieta controle.

Entre os fatores que podem afetar a produção de ovos, destaca-se a ingestão de energia e de proteína pelas aves (Pinto et al., 2002; Freitas et al., 2005). Aparentemente, o comprometimento da digestibilidade da porção nitrogenada da dieta pela inclusão de FACC pode ter contribuído para a queda na produção de ovos.

O peso do ovo diminuiu linearmente com a inclusão de FACC nas rações ( $\left.\hat{\mathrm{Y}}=10,72-0,02 \mathrm{X} ; \mathrm{r}^{2}=12,12 \%\right)$. O peso dos ovos, no entanto, foi significativamente menor, a 5\%

Tabela 2 - Coeficientes de digestibilidade da MS (CDMS), do N (CDN), do EE (CMEE), da EB (CDEB) e valores de energia metabolizável aparente (EMA) e aparente corrigida (EMAn) das dietas

Table 2 - Coefficients of digestibility of DM (DCDM), N (DCN), EE (DCEE) and GE (DCGE) and values of apparent metabolizable energy (AME) and nitrogen corrected apparent metabolizable energy (AMEn) of the diets

\begin{tabular}{|c|c|c|c|c|c|c|c|}
\hline \multirow[t]{2}{*}{ Item } & \multicolumn{6}{|c|}{$\begin{array}{c}\text { Nível de FACC (\%) } \\
\text { CNM level }\end{array}$} & \multirow[t]{2}{*}{ CV (\%) } \\
\hline & 0 & 4 & 8 & 12 & 16 & 20 & \\
\hline CDMS (DCDM) $(\%)^{1}$ & 76,01 & 74,82 & 74,90 & 72,15 & 71,57 & $66,46^{*}$ & 4,815 \\
\hline CDN $(D C N)(\%)^{2}$ & 42,15 & 44,46 & 46,65 & 43,79 & 41,64 & $32,67 *$ & 17,643 \\
\hline CDEE (DCEE) (\%) & 91,77 & 91,36 & 92,12 & 93,14 & 93,63 & 92,37 & 1,50 \\
\hline CDEB (DCCE) (\%) & 81,09 & 80,58 & 80,44 & 78,91 & 79,72 & 77,45 & 3,018 \\
\hline EMA (AME) (kcal/kg MS) & 3,415 & 3,425 & 3,415 & 3,332 & 3,383 & 3,324 & 3,015 \\
\hline EMAn (AMEn) (kcal/kg MS) & 3,315 & 3,320 & 3,301 & 3,227 & 3,285 & 3,246 & 2,641 \\
\hline
\end{tabular}

FACC (farelo de amêndoa da castanha de caju) (CNM, cashew nut meal).

* Diferente em relação ao controle pelo teste Dunnett (5\%)

* Different in relation to control diet by Dunnett test (5\%). 
pelo teste Dunnett, apenas para as aves alimentadas com a dieta com $20 \%$ de FACC.

A menor digestibilidade da proteína com a inclusão do FACC (Tabela 2) pode ter ocasionado menor disponibilidade de aminoácidos para as aves e, conseqüentemente, contribuído para a redução no peso dos ovos. Segundo Buxadé (1993), entre os fatores que podem afetar o peso do ovo, está a ingestão de proteína e aminoácidos, principalmente metionina e lisina.

Apesar de o peso dos ovos ter diminuído com a inclusão de FACC, principalmente no nível de $20 \%$, as médias de peso dos ovos de todos os tratamentos mantiveram-se na faixa considerada normal por Murakami \& Ariki (1998), de 10 a 11 g, com variação de 7 a 14 g. Assim, pode-se afirmar que a inclusão de FACC não compromete a comercialização dos ovos.

A inclusão de FACC na dieta promoveu efeito linear sobre a massa de ovo, que diminuiu conforme aumentaram os níveis de FACC na dieta ( $\hat{Y}=10,17-0,08$ X; $\mathrm{r}^{2}=26,84 \%$ ). Apenas para as aves alimentadas com a dieta com 20\% de FACC, os resultados para massa de ovo foram significativamente menores que os obtidos com a dieta controle (Dunnett 5\%). Considerando que a massa de ovo é calculada multiplicando-se o número de ovos produzidos pelo peso médio dos ovos, esse resultado pode ser atribuído à redução na produção e no peso dos ovos.

A conversão alimentar apresentou aumento linear ( $\left.\hat{Y}=2,22+0,03 X ; r^{2}=36,29 \%\right)$, o que reflete piora no aproveitamento das dietas contendo FACC. Na comparação de médias pelo teste Dunnett, observou-se que, semelhante ao ocorrido para a porcentagem de postura, o peso e a massa de ovo, apenas nas aves alimentadas com a dieta contendo 20\% de FACC, tiveram pior conversão em relação à obtida para as aves alimentadas com a dieta sem FACC.
Diferente do observado nesta pesquisa, Militão (1999), Ojewola et al. (2004) e Freitas et al. (2006) observaram melhora na conversão de frangos de corte alimentados com dietas contendo FACC. Onifade et al. (1998) também verificaram melhor conversão alimentar em frangas de postura em crescimento alimentadas com FACC. Ojewola et al. (2004) atribuíram a melhora na conversão alimentar de frangos de corte ao aumento do teor de gordura nas dietas contendo FACC.

Os resultados das características dos ovos obtidos para os diferentes níveis de FACC nas dietas são apresentados na Tabela 4.

Neste estudo, observou-se efeito quadrático dos níveis de FACC sobre a porcentagem da gema ( $\hat{Y}=30,00-0,18 X$ $\left.+0,01 X^{2} ; r^{2}=35,50 \%\right)$, indicando que a proporção de gema diminuiu inicialmente e voltou a aumentar em níveis superiores a $9 \%$. A porcentagem de albúmen sofreu efeito quadrático dos níveis de FACC na dieta ( $\hat{Y}=61,73+0,16$ $\left.X-0,009 X^{2} ; r^{2}=27,31 \%\right)$, todavia, ao contrário do observado para a gema, a proporção de albúmen nos ovos aumentou inicialmente, atingiu o máximo no nível de 8,8\% de inclusão do alimento e diminuiu em seguida. Os níveis de FACC na dieta não influenciaram $(\mathrm{P}>0,05)$ a porcentagem de casca. Apenas nos ovos das aves alimentadas com dietas contendo $20 \%$ de FACC a porcentagem de gema foi significativamente maior à obtida no tratamento controle, no entanto, a proporção de albúmen foi menor nesses ovos (Dunnett 5\%).

Considerando que a proporção de casca não variou entre os tratamentos e que o aumento da gema foi seguido de redução na proporção de albúmen, pode-se afirmar que o efeito dos níveis de FACC no peso dos ovos deveu-se, em parte, à redução na proporção de albúmen, que não foi superada pelo aumento na proporção de gema.

A ingestão de proteína ou de lisina e metionina podem influenciar o peso do ovo e a proporção de albúmem

Tabela 3 - Desempenho de codornas japonesas alimentadas com dietas contendo diferentes níveis de farelo de amêndoa da castanha de caju (FACC)

Table 3 - $\quad$ Performance of laying quails fed diets containing diferent levels of CNM

\begin{tabular}{lrrrrrr}
\hline Item & \multicolumn{5}{c}{ Nível de FACC (\%) } \\
CNM level
\end{tabular}

FACC (farelo de amêndoa da castanha de caju) (CNM, cashew nut meal).

* Diferente em relação ao controle pelo teste Dunnett (5\%).

* Different in relation to control diet by Dunnett test (5\%). 
Tabela 4 - Características dos ovos de codornas japonesas alimentadas com rações contendo diferentes níveis de FACC Table 4 - Egg characteristics of laying quails fed diets containing diferent levels of CNM

\begin{tabular}{|c|c|c|c|c|c|c|c|}
\hline \multirow[t]{2}{*}{ Item } & \multicolumn{6}{|c|}{$\begin{array}{c}\text { Nível de FACC (\%) } \\
\text { CNM level }\end{array}$} & \multirow[t]{2}{*}{$\mathrm{CV}(\%)$} \\
\hline & 0 & 4 & 8 & 12 & 16 & 20 & \\
\hline Percentagem de albúmen (\%) (Albumen percentage) & 62,54 & 62,31 & 62,21 & 62,37 & 62,09 & $61,16 *$ & 1,05 \\
\hline Porcentagem de casca (\%) (Shell percentage) & 8,47 & 8,31 & 8,40 & 8,29 & 8,27 & 8,17 & 2,84 \\
\hline Cor da gema (Yolk color) & 6,49 & 6,40 & 6,49 & 6,41 & 6,32 & $5,80 *$ & 4,54 \\
\hline
\end{tabular}

FACC (farelo de amêndoa da castanha de caju) (CNM, cashew nut meal).

* Diferente em relação ao controle pelo teste Dunnett (5\%).

* Different in relation to control diet by Dunnett test (5\%).

e gema (Buxadé, 1993). Assim, é possível que a redução na metabolização do nitrogênio, observada principalmente com a inclusão de $20 \%$ do FACC na dieta, tenha interferido na massa e nas características dos ovos produzidos (Tabela 2).

Os níveis de FACC tiveram efeito quadrático ( $\hat{\mathrm{Y}}=6,10$ $+0,09 \mathrm{X}-0,005 \mathrm{X}^{2} ; \mathrm{r}^{2}=41,21 \%$ ) sobre a coloração da gema, que aumentou significativamente, atingiu o máximo com $9 \%$ desse alimento na dieta e diminuiu em seguida. Pela comparação de médias pelo teste Dunnett (5\%), apenas com a inclusão de $20 \%$ de FACC as gemas dos ovos foram menos amarelas que as gemas dos ovos das aves alimentadas com a dieta controle.

Sabe-se que a coloração da gema dos ovos depende da ingestão de pigmentos pelas aves durante a alimentação. Segundo Silva et al. (2000), a substituição total ou parcial do milho por alimentos alternativos pobres em pigmentos carotenóides em dietas para poedeiras pode reduzir a coloração da gema, cuja intensidade depende do nível de substituição. Como pode ser observado na Tabela 1, com a inclusão do FACC, houve redução na quantidade de milho nas dietas. Com isso, certamente a quantidade de pigmentos amarelos reduziu, resultando em gemas menos amarelas.

Apesar da escassez de estudos sobre a preferência do consumidor quanto à coloração da gema de ovos de codornas, esta observação deve ser considerada na formulação de dietas para poedeiras comerciais. Em dietas destinadas à alimentação de aves produtoras de ovos, a inclusão de FACC em níveis acima de $16 \%$ de FACC pode prejudicar a pigmentação das gemas e, nesses casos, deve ser associada ao uso de pigmento.

Com a inclusão de FACC nas dietas em níveis de até $16 \%$, os dados de digestibilidade de nutrientes, de desempenho e características dos ovos não diferiram significativamente dos obtidos com a dieta controle. Desse modo, a inclusão desse alimento em dietas para codornas em postura pode ser feita em níveis de até $16 \%$.

\section{Conclusões}

O farelo da amêndoa da castanha de caju (FACC) pode ser utilizado em níveis de até $16 \%$ na alimentação de codornas japonesas na fase de produção.

\section{Literatura Citada}

BUXADÉ, C.C. El huevo para consumo: bases productivas. Madrid: Mundi Prensa/Adeos, 1993. 401p.

EMPRESA BRASILEIRA DE PESQUISA E AGROPECUÁRIA EMBRAPA. Tabela de composição química e valores energéticos de alimentos para suínos e aves. 3.ed. Concórdia: Embrapa, 1991. p.28-68 (Circular Técnica, 19).

FREITAS E.R.; FUENTES, M.F.F.; SANTOS JR., A. et al. Farelo de castanha de caju em rações para frangos de corte. Pesquisa Agropecuária Brasileira, v.41, n.6, p.1001-1006, 2006.

FREITAS, A.C.; FUENTES, M.F.F.; FREITAS, E.R. et al. Efeito de níveis de proteína bruta e de energia metabolizável na dieta sobre o desempenho de codornas de postura. Revista Brasileira de Zootecnia, v.34, n.3, p.838-846, 2005.

LEESON, S.; SUMMERS, J.D. Nutrition of the chicken, 4 ed Guelph: University Books, 2001. 591p.

MATTERSON, L.D.; POTTER, L. M.; STUTZ, N.W. et al. The metabolizable energy of feed ingredients for chickens. Storrs: The University of Connecticut, Agricultural Experiment Station, 1965. 11p. (Research Report, 7).

MILITÃO, S.F. Utilização do farelo da amêndoa da castanha de caju suplementado com enzimas em dietas de frangos de corte. Fortaleza: Universidade Federal do Ceará, 1999. 113p. Dissertação (Mestrado em Zootecnia) - Universidade Federal do Ceará, 1999.

MURAKAMI, A.E.; FURLAN, A.C. Pesquisa na nutrição e alimentação de codornas em postura no Brasil. In: SIMPOSIO INTERNACIONAL DE COTURNICULTURA, 1., 2002, Lavras. Anais... Lavras: Universidade Federal de Lavras, 2002. p.113-120.

MURAKAMI, A.E.; MORAES, V.M.B.; ARIKI, J. et al. Níveis de proteína e energia em rações para codornas japonesas (Coturnix coturnix japonica) em postura. Revista da Sociedade Brasileira de Zootecnia, v.22, n.4, p.541-551, 1993.

NATIONAL RESEARCH COUNCIL - NRC. Nutrient requirements of poultry. 9.ed. Washington, D.C.: National Academy Press, 1994. 155p.

OJEWOLA, G.S.; OKOYE, F.C.; AGBAKURU, I. Replacement value of cashew-nut meal for soybean meal in finishing broiler chickens. International Journal of Poultry Science, v.3, p.513-516, 2004.

ONIFADE, A.A.; TEWE, O.O.; FANIMO, A.O. et al. Replacement value of cashew nut meal for groundnut-cake in pullet diets: 
Effect on pre-laying performance and serum biochemical indices. Indian Journal Animal Science, v.68, n.3, p.273275, 1998.

ONIFADE, A.A.; TEWE, O.O.; OKUNOLA, O.O. et al. Performance of laying pullets fed on cereal-free diets based on maize offal, cassava peel and reject cashew nut meal. British Poultry Science, v.40, p.84-87, 1999.

PIMENTEL, C.R.M. Castanha de caju: produção e consumo internacional. Fortaleza: EMBRAPA/CNPCA, 1992. 18p.

PINTO, R.; FERREIRA, A.S.; ALBINO, L.F.T. et al. Níveis de proteína e energia para codornas japonesas em postura. Revista Brasileira de Zootecnia, v.31, n.4, p.1761-1770, 2002.

ROSTAGNO, H.S.; ALBINO, L.F.T.; DONZELE, J.L. et al. Composição de alimentos e exigências nutricionais de aves: tabelas brasileiras. Viçosa, MG: Editora UFV, 2000. 141p.
SAKOMURA, N.K.; LONGO, F.A.; RABELLO, C.B. et al. Efeito do nível de energia metabolizável da dieta no desempenho e metabolismo energético de frangos de corte. Revista Brasileira Zootecnia, v.33, p.1758-1767, 2004.

SILVA, D.J.; QUEIROZ, A.C. Análise de alimentos: métodos químicos e biológicos. 3.ed. Viçosa, MG: Editora UFV, 2002. $165 p$.

SILVA, J.H.V.; ALBINO, L.F.T.; GODOI, M.J.S. Efeito do extrato de urucum na pigmentação da gema dos ovos. Revista Brasileira de Zootecnia, v.29, n.5, p.1435-1439, 2000.

STATISTICAL ANALYSIS SYSTEM - SAS. SAS/STAT: user's guide. Version 6, 12.ed. Cary: 2000. (CD-ROM).

Recebido: 05/09/06 Aprovado: 01/03/07 